\title{
Floristic composition and similarity analysis of an Atlantic rain forest fragment in Cananéia, São Paulo State, Brazil ${ }^{1}$
}

\author{
CATIA URBANETZ2,4, JORGE YOSHIO TAMASHIRO³ and LUIZA SUMIKO KINOSHITA ${ }^{3}$
}

(received: September 18, 2008; accepted: October 21, 2010)

\begin{abstract}
Floristic composition and similarity analysis of an Atlantic rain forest fragment in Cananéia, São Paulo State, Brazil). The aim of this study is to investigate the floristic composition of an Atlantic rain forest fragment located in Cananéia, São Paulo, Brazil, and to contribute to the knowledge on Atlantic forest through the comparative analysis of this and other floristic surveys both on the southern and southeastern Brazil, in different soil and relief types. We surveyed 215 species in 132 genera and 51 families. Classification and ordination analysis were applied to a binary matrix in order to analyze the similarity among 24 surveys, including the present one, of Atlantic forest from the south and southeast coast of Brazil. Higher floristic similarity was observed among this area and the ones where there was marine influence and more rugged relief. The surveys in areas with greater marine influence (sandy soil) were separated from those in other conditions, possibly indicating a species replacement gradient from the steep slopes towards the lowland and were probably related to different edaphic conditions. A latitudinal gradient was found among the surveys apparently confirming a continuous species replacement along the Atlantic forest, related to a restricted distribution of the species. This suggests that it is essential to preserve areas from the whole Atlantic coast. Atlantic forest distribution is quite complex and its composition cannot be adequately represented by small localized areas.
\end{abstract}

Key words - lowland rain forest, montane rain forest, shrubs, submontane rain forest, tree community

RESUMO - (Composição florística e análise de similaridade de um trecho de Floresta Ombrófila Densa Atlântica em Cananéia, São Paulo, Brasil). O presente trabalho teve como objetivo caracterizar a flora arbustiva e arbórea de um trecho de Floresta Ombrófila Densa Submontana localizado no Município de Cananéia, São Paulo, de modo a contribuir para o conhecimento da Mata Atlântica através de sua comparação com outros 23 inventários do Sul e Sudeste do Brasil. Foi encontrado um total de 215 espécies distribuídas em 132 gêneros e 51 famílias. Foram realizadas análises de classificação e ordenação aplicadas a uma matriz binária para avaliar a similaridade florística entre os levantamentos. Observou-se maior similaridade entre a área estudada e florestas com menor influência marinha e relevos mais acidentados. Os levantamentos de planície costeira realizados em áreas com influência marinha (solo arenoso) diferenciam-se daqueles feitos em locais com outras condições, indicando um possível gradiente de substituição de espécies da encosta para a planície, que pode estar relacionado com condições edáficas diferenciadas. Foi encontrado um gradiente latitudinal entre os levantamentos analisados, o que parece confirmar uma substituição contínua de espécies ao longo da Mata Atlântica, relacionada com uma distribuição restrita de espécies. Os resultados obtidos sugerem que é essencial a preservação de áreas ao longo de toda a distribuição da Mata Atlântica, uma vez que sua composição florística é bastante complexa e não pode ser adequadamente representada por pequenas áreas.

Palavras-chave - arbustos, comunidade arbórea, Floresta Ombrófila Densa de Terras Baixas, Floresta Ombrófila Densa Montana

\section{Introduction}

The Atlantic forest domain originally extended from the north to the south Brazilian coast with large extensions inland in the Southern and Southeastern regions (Joly et al. 1999, Rizzini 1997). In this domain

1. Part of the first author's MSc Dissertation, Programa de PósGraduação em Biologia Vegetal of Universidade Estadual de Campinas, Campinas, SP, Brazil.

2. Embrapa Pantanal, Rua 21 de setembro 1880, Caixa Postal 109, 79320-900 Corumbá, MS, Brazil.

3. Universidade Estadual de Campinas, Instituto de Biologia, Departamento de Biologia Vegetal, Caixa Postal 6109, 13083-970 Campinas, SP, Brazil.

4. Corresponding author: urbanetz@cpap.embrapa.br different forest physiognomies are still present, occurring east of the dry corridor in northern Brazil, such as Dense Ombrophilous, Open Ombrophilous, Mixed Ombrophilous, Semideciduous and Deciduous Forests, mangroves, restinga, dunes vegetation, estuaries, lagoons and high altitude rocky fields (Joly et al. 1999).

This vegetation has suffered a drastic reduction by human action and only $11.4 \%$ to $16 \%$ of its area remains (Ribeiro et al. 2009) on the steep southern and southeastern slopes. It is considered as one of the biodiversity hotspots by The Conservation International (Myers et al. 2000).

The large continuous areas covered by Atlantic forest in São Paulo State, are concentrated in the southeastern region of Vale do Ribeira. Its relief, climate, 
soil and occupation process have kept it away from the development of the rest of the state, so the forest has been preserved. Several ecosystems of the Atlantic forest still remain in this site (São Paulo 1996).

In the present work we performed the floristic characterization of a fragment of submontane rain forest (Floresta Ombrófila Densa Submontana according to Veloso et al. 1992) localized in the Vale do Ribeira, on the continental part of Cananéia municipality, São Paulo State. This particular area is in a transitional zone of vegetation, relief and soil types. Our objective is to contribute to the knowledge of Atlantic rain forest through the comparative analysis of this and other floristic surveys on the southern and southeastern Brazil, on different soil and relief types. Therefore we aimed to answer the following questions:

1. How could the remnant be characterized? Is its woody flora closer to those which occur on the coastal plain and where there is more marine influence (sandy soil) or to those with less marine influence and more rugged relief (steep slope forests)? 2. Are the floristic samples in coastal areas of the Atlantic forest similar? Are there any obvious gradients or evidence of heterogeneities in this vegetation?

\section{Material and methods}

Study site - The floristic survey was carried out in a submontane rain forest remnant (Floresta Ombrófila Densa Submontana according to Veloso et al. 1992) with about 63 ha in a steep slope that belongs to Folha Larga farm, a private property where the main economic activity is fish farming. Folha Larga farm is located in the continental part of Cananéia municipality, route SP-226, km 31 (245ㄴ' S; 47 $56^{\prime}$ ' W), on Vale do Rio Ribeira, southeast of São Paulo State. The area is in a transitional zone of relief, soil and vegetation types. About $1 \mathrm{~km}$ from the farm there is a continuous remnant of lowland rain forest (Floresta Ombrófila Densa de Terras Baixas according to Veloso et al. 1992) on sandy soil and plain relief. According to the farm owners, 40 years ago the lowest areas (altitude $<60 \mathrm{~m}$ ) of the remnant were used as pasture. They were then abandoned and went through a natural regeneration process. The highest areas (between 60 and $157 \mathrm{~m}$ ) did not suffer any severe interference because of their difficult access. The canopy is about $25 \mathrm{~m}$, with emergent trees $35 \mathrm{~m}$ high. The altitude ranges from 30 to $157 \mathrm{~m}$. The climate type is AF - tropical humid (Koeppen 1948). The climatic data (obtained from the owners) were: annual average temperature $23.4^{\circ} \mathrm{C}$, average temperature of the warmest month $30.6^{\circ} \mathrm{C}$, and the coldest $19.8^{\circ} \mathrm{C}$. The coldest temperature registered was $9.0^{\circ} \mathrm{C}$ and the highest $37.0^{\circ} \mathrm{C}$. The annual precipitation was $1973.58 \mathrm{~mm}$ with a monthly average of $164.47 \mathrm{~mm}$. The soil type is Alisol (Embrapa 2006).
Data collection - Trees and shrubs were collected for the floristic survey through assystematic sampling along existing trails (May-2003 to June-2004). Only individuals higher than $1.5 \mathrm{~m}$ and/or with the first branch higher than $0.5 \mathrm{~m}$ were considered as trees, and as shrubs those that were equal or lower than $1.5 \mathrm{~m}$ and/or with the first branch equal or lower than $0.5 \mathrm{~m}$. The vouchers were prepared by usual methods of plant taxonomy and incorporated to UEC herbarium of Universidade Estadual de Campinas.

Analysis of data - The tree flora of this survey was compared with other 23 in Paraná, São Paulo and Rio de Janeiro States (figure 1). Surveys that fulfilled the following criteria were compared: tree surveys located in coastal plains, tabuleiros, low hills or steep slopes areas in both south and southeast Brazil. At least $80 \%$ of the taxa sampled should be identified at the species level. Only the binomials identified to species and updated according to recent revisions such as Wanderley et al. (2002, 2003, 2005, 2007, 2009), Pennington (1990, 1997), Martins et al. (1996) were considered. The names of the families and species from this survey and other samples

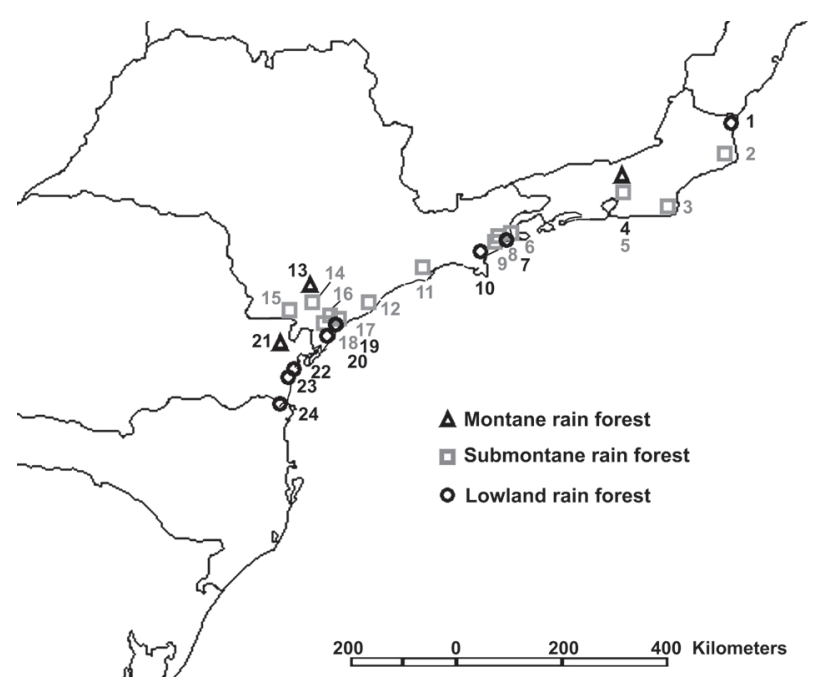

Figure 1. Localization map of the Atlantic Rain forest surveys compared with the present survey. (1 O = Mata do CarvãoRJ (MCa), $2 \square=$ Imbé-RJ (Imb), $3 \square=$ Macacu-RJ (Mac), $4 \Delta=$ Tinguá-RJ (Tin), $5 \square=$ Capoeira Grande-RJ (CGr), $6 \square=$ Picinguaba2-SP (Pic2), $70=$ Picinguaba1-SP (Pic1), $8 \square=$ Picinguaba3-SP (Pic3), $9 \square=$ Ubatuba-SP (Uba), 100 = Caraguatatuba-SP (Car), $11 \square=$ Cubatão-SP (Cub), $12=$ Juréia-SP (Jur), $13 \triangle=$ Parque Estadual de Carlos Botelho1-SP (CBo1), $14 \square=$ Parque Estadual de Carlos Botelho2-SP (CBo2), $15 \square=$ PETAR-SP (PET), $16 \square=$ Pariquera-Açu-SP (PAç), $17 \square=$ Campina do Encantado-SP (CEn), $18 \square=$ Folha Larga-SP (FLa; this survey), $19 \mathrm{O}=$ Ilha do Cardoso 1-SP (ICa1), $200=$ Ilha do Cardoso2-SP (ICa2), $21 \Delta=$ MorretesPR (Mor), $22 \mathrm{O}=$ Paranaguá-PR (Par), $23 \mathrm{O}=$ Ilha do Mel-PR (IMe), $24 \mathrm{O}=$ Volta Velha-SC (VVe)). The localities codes are in tables 2 and 3. (RJ = Rio de Janeiro State; SP = São Paulo State; $\mathrm{PR}=$ Paraná State; $\mathrm{SC}=$ Santa Catarina State). 
were standardized according to APG II (APG 2003), Plant Names Project (2004) and Solomon (2004), and the authors' names according to Brummit \& Powell (1992).

Classification (cluster) and ordination analyses were applied to a matrix of binary data. Cluster was based on Jaccard $\left(\mathrm{J}_{\mathrm{c}}\right)$ index (Mueller-Dombois \& Ellenberg 1974), with arithmetic average (UPGMA), single and complete linkage as clustering methods, to verify the similarity and find possible heterogeneities among the surveys. A Correspondence Analysis (CA, Benzecri 1992) was also performed to detect floristic patterns or gradients and a Detrended Correspondence Analysis (Hill \& Gaugh 1980) in the eventual presence of an arch effect of CA. All analyses were performed using FITOPAC2.1 program (Shepherd \& Urbanetz 2010).

\section{Results and discussion}

Floristic survey - A total of 215 species were found in 132 genera and 51 families, of which seven were palms, 31 shrubs and 177 trees (table 1). Six morphospecies were registered only at genus level (3\% of the surveyed species).

Table 1. Families and species surveyed in the Atlantic Rain forest fragment located in Folha Larga farm, Cananéia, SP $(\mathrm{HIN}=$ UEC herbarium identity number; N.C.F. $=$ not collected fertile).

\begin{tabular}{|c|c|c|}
\hline Family/Specie & Habit & HIN \\
\hline \multicolumn{3}{|l|}{ ANNONACEAE } \\
\hline Guatteria australis A. St.-Hil. & Tree & 133.508 \\
\hline Rollinia sericea (R. E. Fr.) R. E. Fr. & Tree & 132.878 \\
\hline Xylopia brasiliensis Spreng. & Tree & 132.874 \\
\hline Xylopia langsdorffiana Spreng. & Tree & N.C.F. \\
\hline \multicolumn{3}{|l|}{ APOCYNACEAE } \\
\hline $\begin{array}{l}\text { Aspidosperma olivaceum Müll. } \\
\text { Arg. }\end{array}$ & Tree & 133.534 \\
\hline Malouetia arborea (Vell.) Miers & Tree & 132.839 \\
\hline \multicolumn{3}{|l|}{ AQUIFOLIACEAE } \\
\hline Ilex amara (Vell.) Loes. & Tree & 133.710 \\
\hline Ilex theezans Mart. & Tree & 132.849 \\
\hline \multicolumn{3}{|l|}{ ARALIACEAE } \\
\hline Dendropanax monogynum Seem. & Tree & 133.510 \\
\hline $\begin{array}{l}\text { Schefflera angustissima } \\
\text { (Marchal) Frodin }\end{array}$ & Tree & - \\
\hline \multicolumn{3}{|l|}{ ARECACEAE } \\
\hline $\begin{array}{l}\text { Astrocaryum aculeatissimum } \\
\text { (Schott) Burret }\end{array}$ & Palm & - \\
\hline Attalea dubia (Mart.) Burret & Palm & N.C.F \\
\hline $\begin{array}{l}\text { Bactris hatchsbachii Noblick ex } \\
\text { A. J. Hend. }\end{array}$ & Palm & - \\
\hline Bactris setosa Mart. & Palm & N.C.F \\
\hline Euterpe edulis Mart. & Palm & $\begin{array}{l}\text { N.C.F } \\
\text { continu }\end{array}$ \\
\hline
\end{tabular}

continuation

\begin{tabular}{|c|c|c|}
\hline Family/Specie & Habit & HIN \\
\hline Geonoma gamiova Barb. Rodr. & Palm & - \\
\hline $\begin{array}{l}\text { Syagrus romanzoffiana (Cham.) } \\
\text { Glassman }\end{array}$ & Palm & N.C.F \\
\hline \multicolumn{3}{|l|}{ ASTERACEAE } \\
\hline Baccharis semiserrata DC. & Tree & 133.362 \\
\hline $\begin{array}{l}\text { Baccharis singularis (Vell.) G. } \\
\text { M. Barroso }\end{array}$ & Shrub & 133.363 \\
\hline $\begin{array}{l}\text { Pitocarpha macropoda (DC.) } \\
\text { Baker }\end{array}$ & Tree & 132.877 \\
\hline $\begin{array}{l}\text { Vernonia argyrotrichia Sch. Bip. } \\
\text { ex Baker }\end{array}$ & Shrub & - \\
\hline Vernonia beyrichii Less. & Shrub & - \\
\hline Vernonia petiolaris DC. & Tree & 132.886 \\
\hline Vernonia puberula Less. & Shrub & 133.368 \\
\hline $\begin{array}{l}\text { Vernonia rubriramea Mart. ex } \\
\text { DC. }\end{array}$ & Shrub & - \\
\hline \multicolumn{3}{|l|}{ BIGNONIACEAE } \\
\hline Jacaranda puberula Cham. & Tree & 132.842 \\
\hline $\begin{array}{l}\text { Tabebuia umbellata (Sond.) } \\
\text { Sandwith }\end{array}$ & Tree & 133.529 \\
\hline \multicolumn{3}{|l|}{ BORAGINACEAE } \\
\hline Cordia magnoliifolia Cham. & Tree & - \\
\hline $\begin{array}{l}\text { Cordia sellowiana Cham. } \\
\text { BURSERACEAE }\end{array}$ & Tree & 132.867 \\
\hline $\begin{array}{l}\text { Protium heptaphyllum (Aubl.) } \\
\text { Marchand }\end{array}$ & Tree & 132.888 \\
\hline \multicolumn{3}{|l|}{ CELASTRACEAE } \\
\hline \multicolumn{2}{|l|}{ CHRYSOBALANACEAE } & 133.925 \\
\hline Hirtella hebeclada Moric. ex DC. & Tree & 133.541 \\
\hline $\begin{array}{l}\text { Licania octandra (Hoffmanns. ex } \\
\text { Roem. \& Schult.) Kuntze }\end{array}$ & Tree & 132.866 \\
\hline Parinari excelsa Sabine & Tree & 133.557 \\
\hline \multicolumn{3}{|l|}{ CLETHRACEAE } \\
\hline \multicolumn{2}{|l|}{ CLUSIACEAE } & - \\
\hline Calophyllum brasiliense Cambess. & Tree & N.C.F \\
\hline Clusia criuva Cambess. & Tree & 132.887 \\
\hline $\begin{array}{l}\text { Garcinia gardneriana (Planch. \& } \\
\text { Triana) Zappi }\end{array}$ & Tree & 133.525 \\
\hline \multicolumn{3}{|l|}{ CUNONIACEAE } \\
\hline $\begin{array}{l}\text { Weinmannia paulliniifolia Pohl } \\
\text { ex Ser. }\end{array}$ & Tree & - \\
\hline \multicolumn{3}{|l|}{ ELAEOCARPACEAE } \\
\hline $\begin{array}{l}\text { Sloanea guianensis (Aubl.) Benth. } \\
\text { ERYTHROXYLACEAE }\end{array}$ & Tree & 132.889 \\
\hline $\begin{array}{l}\text { Erythroxylum cuspidifolium Mart. } \\
\text { EUPHORBIACEAE }\end{array}$ & Tree & 133.540 \\
\hline $\begin{array}{l}\text { Actinostemon concolor (Spreng.) } \\
\text { Müll. Arg }\end{array}$ & Tree & 133.905 \\
\hline Alchornea glandulosa Poepp. & Tree & $\begin{array}{l}132.829 \\
\text { continuc }\end{array}$ \\
\hline
\end{tabular}


continuation

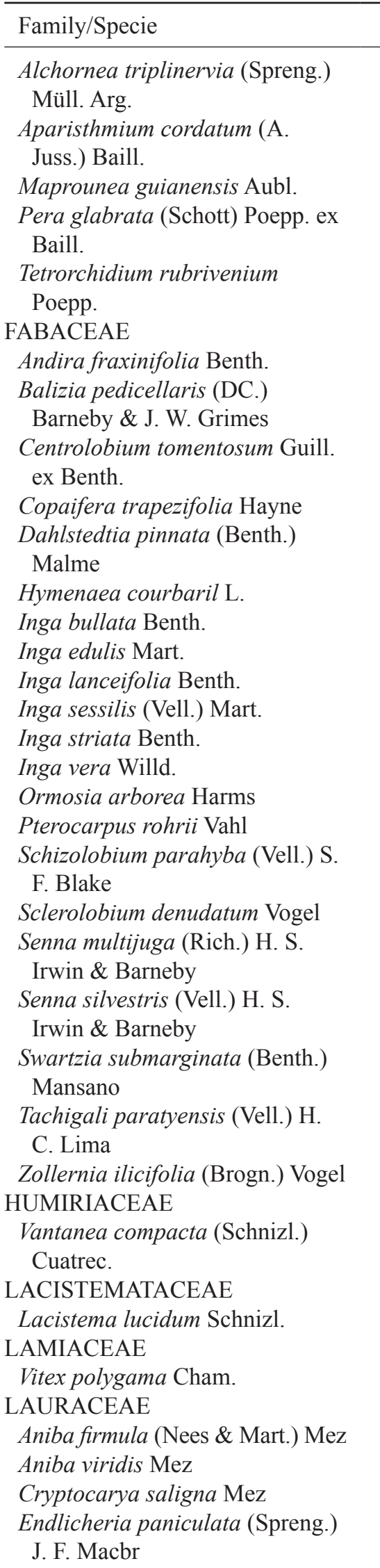

continuation

\begin{tabular}{|c|c|c|}
\hline Family/Specie & Habit & HIN \\
\hline $\begin{array}{l}\text { Licaria armeniaca }(\text { Nees) } \\
\text { Kosterm. }\end{array}$ & Tree & 133.543 \\
\hline Nectandra aff. nitidula Nees & Tree & N.C.F \\
\hline $\begin{array}{l}\text { Nectandra oppositifolia } \text { Nees \& } \\
\text { Mart. }\end{array}$ & Tree & 133.898 \\
\hline Ocotea aciphylla (Nees) Mez & Tree & - \\
\hline Ocotea brachybotra Mez & Tree & N.C.F \\
\hline Ocotea daphnifolia (Meisn.) Mez & Tree & 133.706 \\
\hline $\begin{array}{l}\text { Ocotea diospyrifolia (Meisn.) } \\
\text { Mez }\end{array}$ & Tree & 133.372 \\
\hline Ocotea dispersa $\mathrm{Mez}$ & Tree & N.C.F \\
\hline Ocotea elegans $\mathrm{Mez}$ & Tree & N.C.F \\
\hline Ocotea nectandrifolia $\mathrm{Mez}$ & Tree & 133.371 \\
\hline Ocotea silvestris Vattimo-Gil & Tree & 132.830 \\
\hline Ocotea venulosa (Ness) Baitello & Tree & N.C.F \\
\hline $\begin{array}{l}\text { Rhodostemonodaphne } \\
\text { macrocalyx (Meisn.) Rohwer ex } \\
\text { Madriñán }\end{array}$ & Tree & 133.370 \\
\hline \multicolumn{3}{|l|}{ LECYTHIDACEAE } \\
\hline $\begin{array}{l}\text { Cariniana estrellensis (Raddi) } \\
\text { Kuntze }\end{array}$ & Tree & N.C.F \\
\hline MALPIGHIACEAE & & \\
\hline $\begin{array}{l}\text { Byrsonima ligustrifolia A. Juss. } \\
\text { MALVACEAE }\end{array}$ & Tree & 132.892 \\
\hline Pseudobombax grandiflorum & Tree & - \\
\hline
\end{tabular}

(Cav.) A. Robyns

MELASTOMATACEAE

Leandra australis Cogn.

Leandra melastomoides Raddi

Leandra scabra DC.

Leandra cf. dasytricha (A. Gray) Cogn.

Tree _ - _ Leandra cf. dubia DC

Leandra cf. nianga Cogn.

Tree - $\quad$ Miconia cabucu Hoehne

Tree N.C.F

Tree N.C.F

Tree

Tree $\quad 133.903$

Tree $\quad 133.494$

Tree $\quad 133.512$

Tree N.C.F

Tree $\quad 133.707$

Tree N.C.F

continue
Miconia cinerascens Miq.

Naudin

Miconia cubatanensis Hoehne Tree

Miconia dodecandra Cogn. Tree

Miconia hymenonervia (Raddi) Tree

Cogn.

Miconia rigidiuscula Cogn.

Miconia saldanhaei Cogn.

Ossaea brachystachya Triana

Tibouchina arborea Cogn.

Tibouchina mutabilis Cogn.

Tibouchina weddelii Cogn.

MELIACEAE

Cabralea canjerana (Vell.) Mart. Tree

Cedrela fissilis Vell.

Guarea macrophylla Vahl
Miconia cinnamomifolia (DC.)
Shrub

Shrub

Shrub

Tree

Shrub

Shrub

Tree

Tree

Tree

133.476

133.361

-

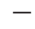

$-$

Tree

Shrub

Shrub

133.475

Tree

133.478

Tree

Tree

132.873

Tree

133.552

Tree
132.831 continue 
continuation

\begin{tabular}{|c|c|c|}
\hline Family/Specie & Habit & HIN \\
\hline \multicolumn{3}{|l|}{ MONIMIACEAE } \\
\hline $\begin{array}{l}\text { Mollinedia schottiana (Spreng.) } \\
\text { Perkins }\end{array}$ & Shrub & 133.496 \\
\hline \multicolumn{2}{|l|}{ MORACEAE } & 132.841 \\
\hline Brosimum glaziovii Taub. & Tree & - \\
\hline $\begin{array}{l}\text { Brosimum guianensis (Aubl.) } \\
\text { Huber }\end{array}$ & Tree & - \\
\hline Ficus pulchella Schott ex Spreng. & Tree & 133.373 \\
\hline Sorocea bonplandii Baill. & Tree & N.C.F \\
\hline \multicolumn{3}{|l|}{ MYRISTICACEAE } \\
\hline $\begin{array}{l}\text { Virola bicuhyba (Schott ex } \\
\text { Spreng.) Warb. }\end{array}$ & Tree & 132.868 \\
\hline $\begin{array}{l}\text { Virola gardneri (A. DC.) Warb. } \\
\text { MYRSINACEAE }\end{array}$ & Tree & 133.941 \\
\hline Ardisia guianensis (Aubl.) Mez & Tree & 133.532 \\
\hline $\begin{array}{l}\text { Myrsine ferruginea (Ruiz \& Pav.) } \\
\text { Spreng. }\end{array}$ & Tree & 133.708 \\
\hline $\begin{array}{l}\text { Myrsine guianensis (Aubl.) } \\
\text { Kuntze }\end{array}$ & Tree & 132.847 \\
\hline \multicolumn{3}{|l|}{ MYRTACEAE } \\
\hline $\begin{array}{l}\text { Blepharocalyx salicifolius } \\
\text { (Kunth) O. Berg. }\end{array}$ & Shrub & 133.516 \\
\hline $\begin{array}{l}\text { Calyptranthes fusiformis M. L. } \\
\text { Kawas. }\end{array}$ & Tree & 133.723 \\
\hline Calyptranthes grandifolia $\mathrm{O}$. Berg & Tree & 133.722 \\
\hline Calyptranthes lucida Mart. ex DC. & Tree & 133.517 \\
\hline Calyptranthes strigipes O. Berg & Tree & - \\
\hline $\begin{array}{l}\text { Campomanesia guaviroba (DC.) } \\
\text { Kiaersk. }\end{array}$ & Tree & 133.725 \\
\hline Eugenia acutata Miq. & Tree & 133.731 \\
\hline Eugenia bocainensis Mattos & Shrub & 133.726 \\
\hline Eugenia brasiliensis Lam. & Tree & 133.963 \\
\hline Eugenia copacabanensis Kiaersk. & Tree & 133.962 \\
\hline Eugenia cuprea Nied. & Shrub & 133.486 \\
\hline $\begin{array}{l}\text { Eugenia melanogyna }(\mathrm{D} . \\
\text { Legrand) Sobral }\end{array}$ & Tree & 133.729 \\
\hline Eugenia mosenii (Kausel) Sobral & Tree & 133.732 \\
\hline Eugenia multicostata D. Legrand & Tree & 133.727 \\
\hline Eugenia neoaustralis Sobral & Tree & - \\
\hline Eugenia umbelliflora O. Berg & Tree & - \\
\hline Gomidesia flagellaris D. Legrand & Tree & 133.716 \\
\hline Gomidesia schaueriana O. Berg & Tree & 133.720 \\
\hline $\begin{array}{l}\text { Gomidesia spectabilis (DC.) O. } \\
\text { Berg }\end{array}$ & Tree & - \\
\hline $\begin{array}{l}\text { Gomidesia tijucensis (Kiaersk.) } \\
\text { D. Legrand }\end{array}$ & Tree & - \\
\hline $\begin{array}{l}\text { Marlierea eugeniopsoides (D. } \\
\text { Legrand \& Kausel) D. Legrand }\end{array}$ & Tree & 133.956 \\
\hline Marlierea tomentosa Cambess. & Tree & 133.721 \\
\hline Myrcia heringii D. Legrand & Shrub & - \\
\hline $\begin{array}{c}\text { Myrcia multiflora }(\text { Lam.) DC. } \\
\text { continue }\end{array}$ & Tree & 133.966 \\
\hline
\end{tabular}

continuation

\begin{tabular}{|c|c|c|}
\hline Family/Specie & Habit & HIN \\
\hline Myrcia pubipetala Miq. & Tree & 133.969 \\
\hline Myrcia splendens (Sw.) DC. & Tree & 132.855 \\
\hline $\begin{array}{l}\text { Myrcia cf. isaiana G. M. Barroso } \\
\text { \& Peixoto }\end{array}$ & Shrub & 132.882 \\
\hline $\begin{array}{l}\text { Myrcia stictophylla (O. Berg) N. } \\
\text { J. E. Silveira }\end{array}$ & Tree & 133.968 \\
\hline $\begin{array}{l}\text { Psidium cattleianum Sabine } \\
\text { NYCTAGINACEAE }\end{array}$ & NYCTAGINACEAE & 133.728 \\
\hline Guapira opposita (Vell.) Reitz & Tree & 133.498 \\
\hline Neea schwackeana Heimerl & Shrub & 133.535 \\
\hline $\begin{array}{l}\text { OCHNACEAE } \\
\text { Ouratea parviflora (DC.) Baill. }\end{array}$ & Tree & 133.938 \\
\hline Heisteria silvianii Schwacke & Tree & 132.871 \\
\hline $\begin{array}{l}\text { Tetrastylidium grandifolium } \\
\text { (Baill.) Sleumer }\end{array}$ & Tree & 133.553 \\
\hline \multicolumn{3}{|l|}{ PHYLLANTHACEAE } \\
\hline Hyeronima alchorneoides Allemão & Tree & 133.536 \\
\hline Richeria grandis Vahl & Tree & 132.863 \\
\hline \multicolumn{3}{|l|}{ PIPERACEAE } \\
\hline Ottonia martiana Miq. & Shrub & 133.554 \\
\hline Piper cernuит Vell. & Shrub & 133.944 \\
\hline Piper aduncum L. & Shrub & 133.953 \\
\hline \multicolumn{3}{|l|}{ POLYGONACEAE } \\
\hline Coccoloba mosenii Lindau & Tree & 132.852 \\
\hline Coccoloba sp. & Tree & N.C.F \\
\hline \multicolumn{3}{|l|}{ QUIINACEAE } \\
\hline Quiina glaziovii Engl. & Tree & N.C.F \\
\hline \multicolumn{3}{|l|}{ ROSACEAE } \\
\hline \multicolumn{2}{|l|}{ RUBIACEAE } & 132.885 \\
\hline Amaioua intermedia Mart. & Tree & N.C.F \\
\hline Bathysa australis K. Schum. & Tree & N.C.F \\
\hline $\begin{array}{l}\text { Chomelia catharinae (L. B. Sm. } \\
\text { \& Downs) Steyerm. }\end{array}$ & Shrub & 133.524 \\
\hline $\begin{array}{l}\text { Faramea montevidensis (Cham. } \\
\text { \& Schltdl.) DC. }\end{array}$ & Tree & 133.358 \\
\hline $\begin{array}{l}\text { Faramea multiflora A. Rich. ex } \\
\text { DC. }\end{array}$ & Tree & 133.380 \\
\hline Ixora heterodoxa Müll. Arg. & Tree & 133.523 \\
\hline $\begin{array}{l}\text { Posoqueria latifolia (Rudge) } \\
\text { Roem. \& Schult. }\end{array}$ & Tree & 133.489 \\
\hline $\begin{array}{l}\text { Psychotria birotula L. B. Sm. \& } \\
\text { Downs }\end{array}$ & Shrub & 133.378 \\
\hline Psychotria carthargenensis Jacq. & Tree & - \\
\hline Psychotria gracilenta Müll. Arg. & Shrub & - \\
\hline $\begin{array}{l}\text { Psychotria hoffmannseggiana } \\
\text { Müll. Arg. }\end{array}$ & Shrub & 132.860 \\
\hline Psychotria laciniata Vell. & Tree & 133.558 \\
\hline $\begin{array}{l}\text { Psychotria leiocarpa Cham. \& } \\
\text { Schltdl. }\end{array}$ & Tree & 133.375 \\
\hline $\begin{array}{c}\text { Psychotria mapourioides DC. } \\
\text { continue }\end{array}$ & Tree & 132.891 \\
\hline
\end{tabular}


continuation

\begin{tabular}{|c|c|c|}
\hline Family/Specie & Habit & HIN \\
\hline $\begin{array}{l}\text { Psychotria nuda (Cham. \& } \\
\text { Schltdl.) Wawra }\end{array}$ & Tree/Shrub & - \\
\hline Rudgea recurva Müll. Arg. & Tree & 133.383 \\
\hline \multicolumn{3}{|l|}{ RUTACEAE } \\
\hline Esenbeckia grandiflora Mart. & Tree & 132.869 \\
\hline Zanthoxylum rhoifolium Lam. & Tree & 133.561 \\
\hline \multicolumn{3}{|l|}{ SALICACEAE } \\
\hline Casearia obliqua Spreng. & Tree & N.C.F \\
\hline Casearia sylvestris Sw. & Tree & 133.715 \\
\hline \multicolumn{3}{|l|}{ SAPINDACEAE } \\
\hline Cupania oblongifolia Camb. & Tree & 133.902 \\
\hline Matayba guyanensis Aubl. & Tree & - \\
\hline Matayba intermedia Radlk. & Tree & 133.556 \\
\hline Matayba sp. & Tree & N.C.F \\
\hline \multicolumn{3}{|l|}{ SAPOTACEAE } \\
\hline Chrysophyllum flexuosum Mart. & Tree & 133.354 \\
\hline $\begin{array}{l}\text { Diploon cuspidatum (Hoehne) } \\
\text { Cronquist }\end{array}$ & Tree & 133.497 \\
\hline Ecclinusa ramiflora Mart. & Tree & 133.493 \\
\hline $\begin{array}{l}\text { Manilkara subsericea (Mart.) } \\
\text { Dubard }\end{array}$ & Tree & - \\
\hline $\begin{array}{l}\text { Pouteria beaurepairei (Glaz. \& } \\
\text { Raunk.) Baehni }\end{array}$ & Tree & - \\
\hline Pouteria caimito Radlk. & Tree & - \\
\hline \multicolumn{3}{|l|}{ SOLANACEAE } \\
\hline $\begin{array}{l}\text { Brunfelsia pauciflora (Cham. \& } \\
\text { Schltdl.) Benth. }\end{array}$ & Shrub & 133.342 \\
\hline $\begin{array}{l}\text { Cestrum sessiliflorum Schott ex } \\
\text { Sendt }\end{array}$ & Shrub & 133.531 \\
\hline Solanum pseudo-quina A. St.-Hil. & Tree & 133.555 \\
\hline Solanum undulatum Dunal & Shrub & 132.837 \\
\hline $\begin{array}{l}\text { Solanum swartzianum Roem. \& } \\
\text { Schultz }\end{array}$ & Tree & 133.488 \\
\hline \multicolumn{3}{|l|}{ SYMPLOCACEAE } \\
\hline Symplocos laxiflora Benth. & Tree & 133.520 \\
\hline $\begin{array}{l}\text { Symplocos variabilis Mart. ex Miq. } \\
\text { THYMELAEACEAE }\end{array}$ & Tree & 132.890 \\
\hline $\begin{array}{l}\text { Daphnopsis schwackeana Taub. } \\
\text { URTICACEAE }\end{array}$ & Shrub & 132.850 \\
\hline Cecropia glaziovi Snethl. & Tree & - \\
\hline Cecropia pachystachia Trécul & Tree & - \\
\hline $\begin{array}{l}\text { Coussapoa microcarpa (Schott.) } \\
\text { Rizzini }\end{array}$ & Tree & 132.864 \\
\hline $\begin{array}{l}\text { Pourouma guyanensis Aubl. } \\
\text { VERBENACEAE }\end{array}$ & Tree & 133.932 \\
\hline Aegiphila sellowiana Cham. & Tree & N.C.F \\
\hline $\begin{array}{l}\text { Citharexylum myrianthum Cham. } \\
\text { VOCHYSIACEAE }\end{array}$ & Tree & 133.500 \\
\hline $\begin{array}{l}\text { Callisthene kuhlmmanii H. F. } \\
\text { Martins }\end{array}$ & Tree & 133.940 \\
\hline Vochysia bifalcata Warm. & Tree & 133.943 \\
\hline
\end{tabular}

The richest families were Myrtaceae (28 species), Fabaceae (21), Melastomataceae (18), Lauraceae (17), Rubiaceae (16), Asteraceae (9), Arecaceae (7), Euphorbiaceae (7), Sapotaceae (7), and Solanaceae (5) (63\% of total number of species). The other families presented from four to one species.

Those 10 families were among the richest ones on woody floras of 102 Atlantic rain forest surveys of southeast Brazil analyzed by Oliveira Filho \& Fontes (2000). Asteraceae was among the richest families in high altitudes according to these authors, but six of the Asteraceae species found in Folha Larga farm were shrubs, that highlights the importance of collecting plants of other habits besides trees in a forest thus improving the characterization of a site.

The richest genera were Eugenia (10 species), Ocotea (9), Miconia and Psychotria (8), Myrcia (6), Inga, Leandra, and Vernonia (6).

Similarity analysis - The list of the samples analyzed, codes of the samples, references, localities, sample methods, habits, inclusion criteria and vegetation types (according to Veloso et al. 1992) are shown in table 2. The localization map of the surveys is shown in figure 1. Table 3 presents the environmental characteristics of those surveys. Geographic coordinates were obtained from the corresponding municipality where not provided by the authors. A presence/absence matrix was obtained from 24 samples (including the present survey) with 915 binomials, of which 458 were not taken into account because they were present in only one survey.

More than $95 \%$ of the obtained Jaccard's indexes ware $\mathrm{J}_{\mathrm{c}}<0.3$ which indicates a high floristic heterogeneity among the localities. According to this index, the study area FLa (Folha Larga-SP) presents more floristic similarity to PAç (Pariquera-Açu-SP; $\mathrm{J}_{\mathrm{c}}=0.42$ ), Jur (Juréia-SP; $J_{c}=0.37$ ), VVe (Volta Velha-SC; $J_{c}=0.31$ ), CBol (Carlos Botelho 1-SP) and Cub (Cubatão-SP; $\mathrm{J}_{\mathrm{c}}=0.26$ ), ICa2 (Ilha do Cardoso-SP) and CEn (Campina do Encantado-SP; $\left.\mathrm{J}_{\mathrm{c}}=0.25\right)$.

The lowland samples (Floresta Ombrófila Densa de Terras Baixas according to Veloso et al. 1992) of São Paulo and Paraná: Car (Caraguatatuba), ICa1 and ICa2 (Ilha do Cardoso), Pic1 (Picinguaba), IMe (Ilha do Mel), Par (Paranaguá) formed a group in all dendrograms, isolated from those in the steep slopes and low hills (group 2 - figure 2). The survey VVe (coastal plain of Santa Catarina) occupied an intermediate position, near the steep slopes samples in the complete match and in the UPGMA dendrogram (figure 2) and near the lowland surveys in the simple match, which indicate it could bear 


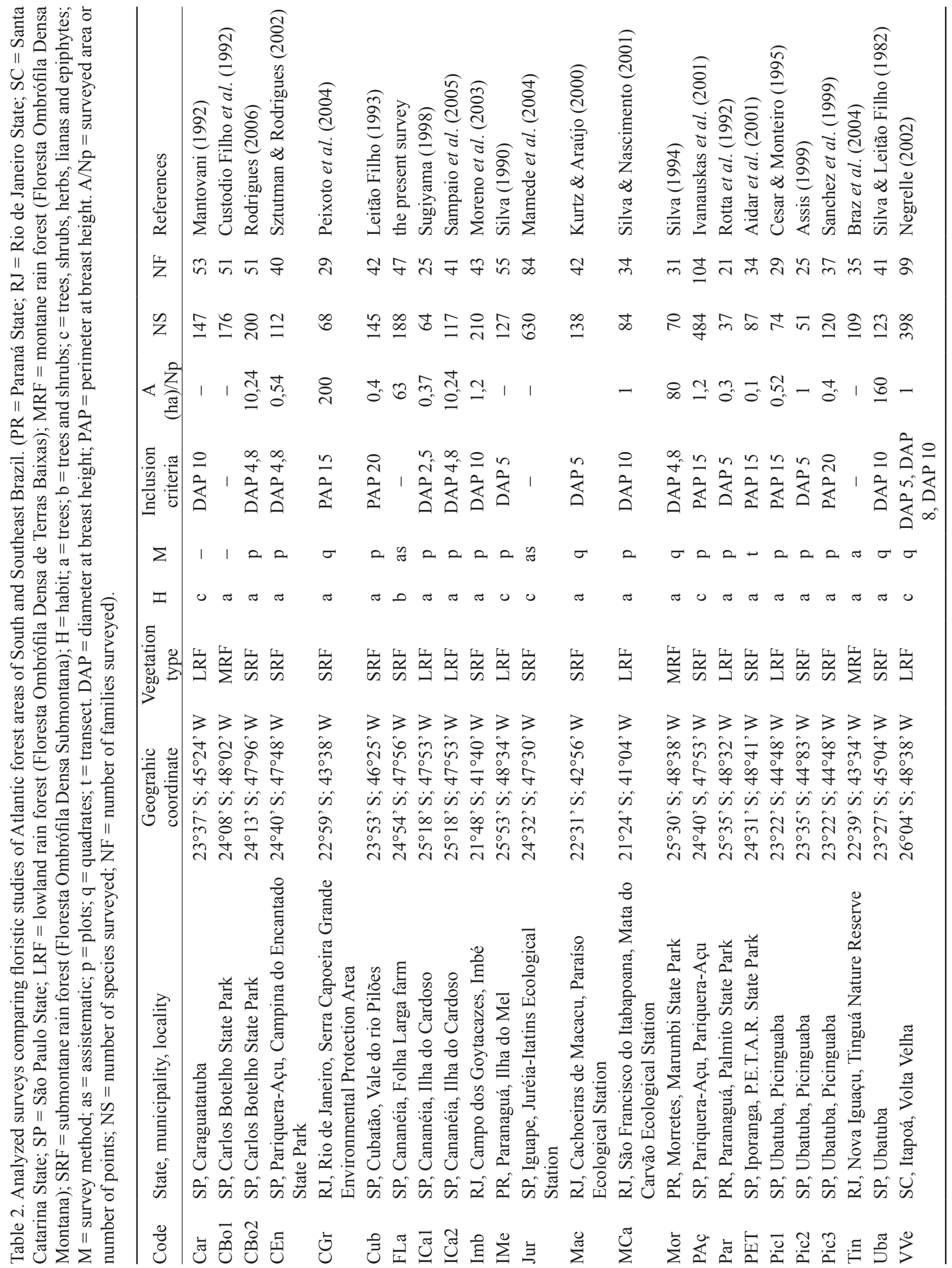




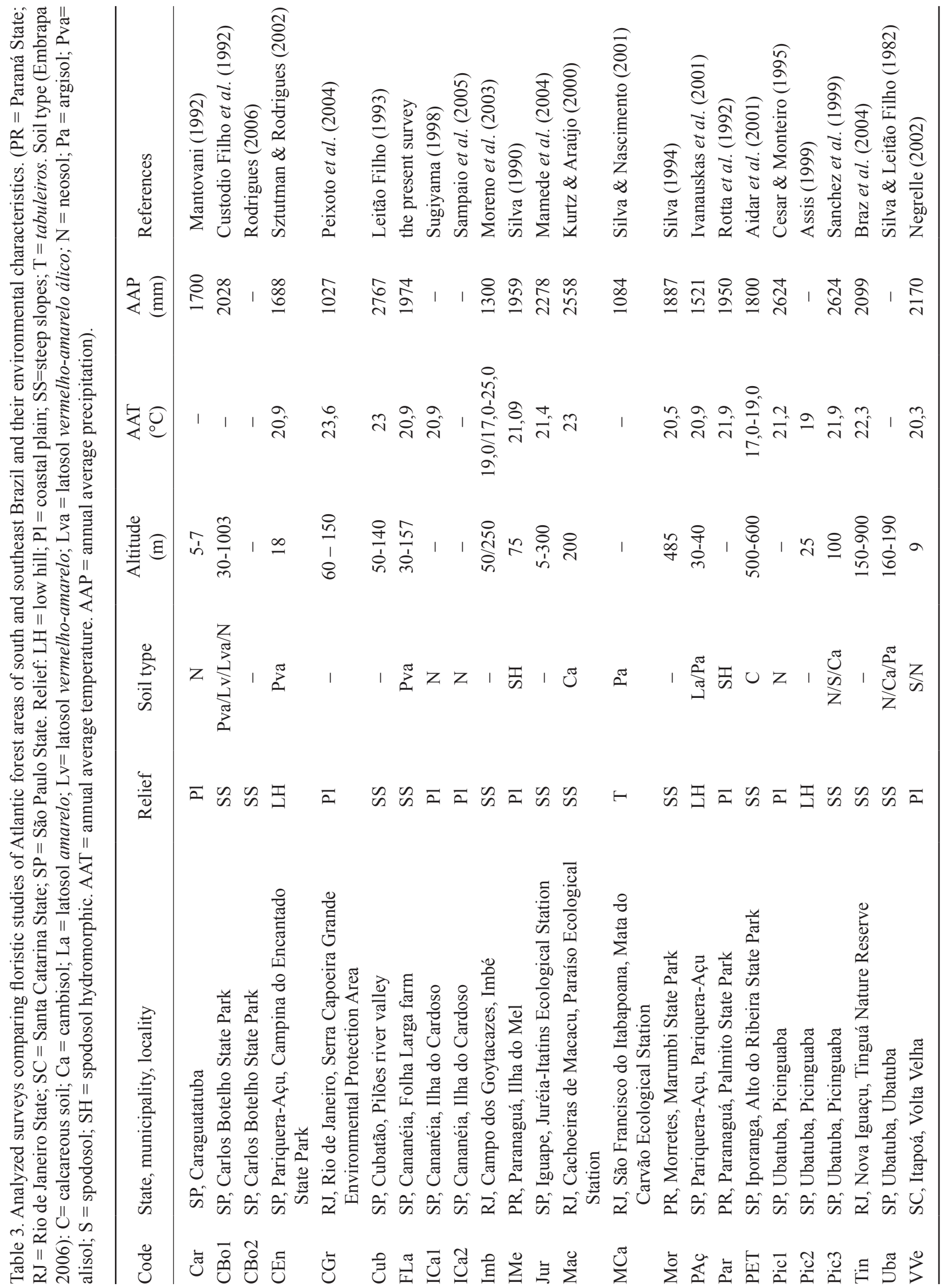




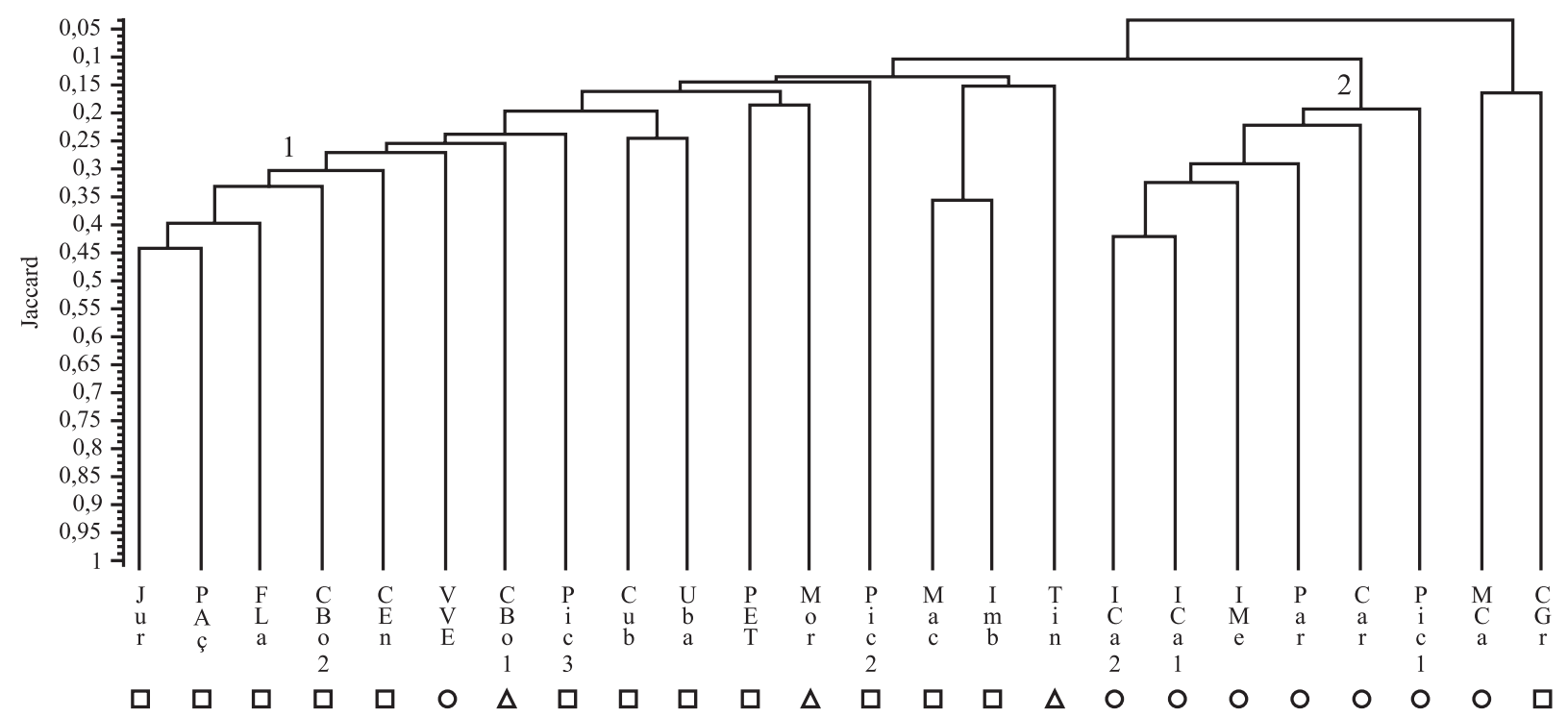

Figure 2. Cluster analysis by Jaccard index and UPGMA group linkage method applied to a binary matrix of tree species surveyed in 24 analyzed areas of Atlantic Rain forest (cofenetic correlation $=0,87$ ). ( $\Delta=$ montane rain forest, $\square=$ submontane rain forest, $O=$ lowland rain forest). The localities codes are in figure 1 and tables 2 and 3 .

a transitional flora. Only the UPGMA dendrogram are presented since it better represented the floristic patterns found in all the analyses.

The samples Jur (Juréia), PAç (Pariquera-Açu), FLa (Folha Larga), CBo2 (Carlos Botelho 2) and CEn (Campina do Encantado) (group 1 - figure 2) and $\mathrm{MCa}$ (Mata do Carvão) and CGr (Capoeira Grande) formed two isolated groups in all dendrograms. The other surveys did not form any floristic pattern, varying their position according to the clustering method applied. In $\mathrm{MCa}$ (Mata do Carvão-RJ) the climate could be a determining factor in the differentiation of its flora because this sample was situated in a tabuleiros area (coastal table land with low altitude) with seasonal climate, and comparatively low annual average precipitation $(1,084 \mathrm{~mm})$. This climatic characteristic is due to the relief in the north of Rio de Janeiro where the mountain chains reach the seacoast, so the rainfall changes in this direction. The CGr (Capoeira Grande) area was surveyed in a coastal plain area with low altitude (60-150 m) and comparatively low annual average precipitation $(1,027 \mathrm{~mm})$.

In all dendrograms, the survey Pic2 (Picinguaba-SP) is more similar to the steep slopes samples of São Paulo and Rio de Janeiro States than to the lowland adjacent sample Pic1 (Picinguaba-SP). That might be explained by the fact that Pic2 sample was located in a low isolated hill, probably with low marine influence on the soil, different from Pic1 in a sandy soil.
An arch was formed in Correspondence Analysis (CA, figure 3), indicating an unimodal distribution of species along an existing gradient. However, all the lowland areas appeared in an extremity of the arch (figure 3), with the samples of Rio de Janeiro State in the opposite end.

Since an arch was found in CA, Detrended Correspondence Analysis (DCA) was performed to

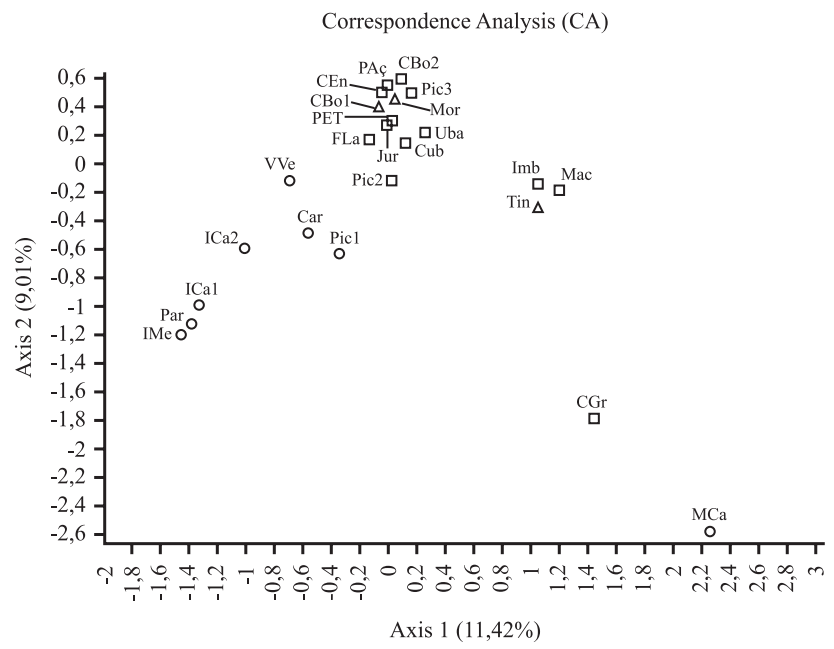

Figure 3. Axis 1 and 2 of Correspondence Analysis (CA) from a binary matrix of tree species surveyed in 24 analyzed areas of Atlantic Rain forest. ( $\Delta=$ montane rain forest, $\square=$ submontane rain forest, $O=$ lowland rain forest). The localities codes are in figure 1 and tables 2 and 3. 
correct this effect. The lowland rain forest samples are separated from submontane and montane rain forest on the left of the first axis of DCA (figure 4), not in a isolated floristic block but in a gradient. A latitudinal gradient could be noted also in the axis 1 . The surveys of Rio de Janeiro State are on the right position in axis 1 and the steep slopes and low hills samples of São Paulo together with the sample Mor (Morretes-PR) are in the middle (figure 4). The survey Pic2 (Picinguaba-SP, coastal plain) is closer to the steep slopes samples than to the adjacent lowland sample Pic1 (Picinguaba-SP) which also confirms the results of cluster analysis.

According to all analyses, the tree flora of Folha Larga farm presents more similarity to the samples collected in the nearest areas with low marine influence, even though the area is located on the edge between a sandy coastal plain and the steep slopes. This confirms the results obtained by Scudeller et al. (2001), where the highest similarities are among samples geographically closer. That could be related to a low proportion of wide distributed species on the Atlantic rain forest.

A latitudinal gradient was found and the surveys of Rio de Janeiro, which were in the north of the analysed samples, were separated from the others. According to Oliveira Filho \& Fontes (2000), annual average temperature is probably the most important factor to north-south differentiation of coastal areas of Atlantic forest. The gradient changes due to temperature decline in this direction. According to Scudeller et al. (2001), besides temperature, altitude is also one of the most important factors related to species distribution. The low similarity among the analyzed samples and the high number of exclusive species (about 50\%) also strengthen the hypothesis of a restrict species distribution in Atlantic forest.

No clear separation was found between the north and the south samples of São Paulo State as Leitão Filho (1982) proposed. The results were in accordance with Scudeller et al. (2001), who observed a long non linear gradient along the Atlantic rain forests of São Paulo, without any distinct floristic blocks. According to these authors this is related to a restricted species distribution in the Atlantic rain forest as $77 \%$ of species occur in less than $20 \%$ of the analyzed surveys in São Paulo.

The lowland rain forest areas (Floresta Ombrófila Densa de Terras Baixas according to Veloso et al. 1992), with the highest marine influence, could be differentiated, in floristic terms, from the submontane and montane rain forest areas in the steep slopes and low hills (Floresta Ombrófila Densa Submontana and Montana, respectively according to Veloso et al. 1992), since in cluster analysis these samples appeared separate from each other. However, this separation was not abrupt to form floristic blocks because of the obvious gradient found in CA

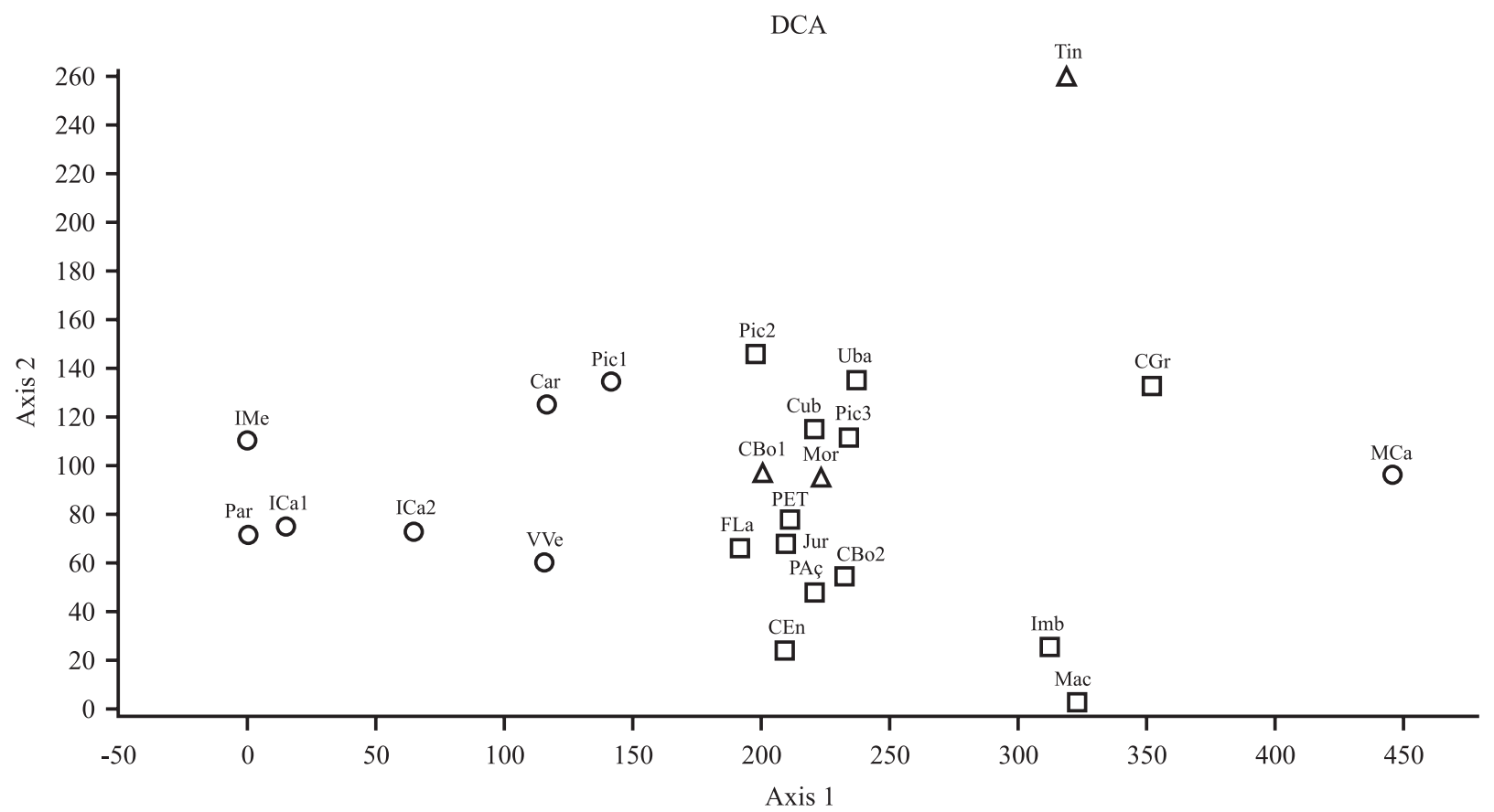

Figure 4. Axis 1 and 2 of Detrended Correspondence Analysis (DCA) from a binary matrix of tree species surveyed in 24 analyzed areas of Atlantic Rain forest. ( $\Delta=$ montane rain forest, $\square=$ submontane rain forest, $O=$ lowland rain forest). The localities codes are in figure 1 and tables 2 and 3 . 
analysis. According to Rizzini (1997) there is probably a species gradient from the steep slopes to the seashore. The results of CA and DCA analysis seem to confirm this observation. The gradient could be related to differential conditions of soil according to its origin. A possible hypothesis is that there are two gradients, an edaphic one in the steep slopes-sea direction, and a latitudinal one in the north-south direction. This division indicates a continuous gradient of species replacement, apparently related to differential edaphic conditions (associated with the degree of marine influence) and the restriction of the species distribution. However, a detailed analysis of the abiotic conditions is necessary, specially of the soil characteristics, to confirm this hypothesis.

Only 13 tree species (less than $10 \%$ of the total) were found just in this survey (exclusive species). The distribution of these exclusive species placed the local area either in the south or north limits of their distribution. The distribution of Ardisia guyanensis (Myrsinaceae) is from North of Brazil to Santa Catarina State (Bernacci \& Jung-Mendaçolli 2000). Bactris hatchsbachii (Arecaceae) occurs in São Paulo and Paraná States and according to Henderson et al. (1995) it is considered a rare and probably endangered species. Callisthene kuhlmannii (Vochysiaceae) occurs in São Paulo, Paraná and Santa Catarina, and the south of São Paulo is its northern limit (Martins 1988). The occurrence of Coccoloba mosenii (Polygonaceae) reaches Bahia, Espírito Santo, Rio de Janeiro and São Paulo States (Melo \& MarcondesFerreira 2009). Cordia magnoliifolia (Boraginaceae) occurs in Rio de Janeiro, Minas Gerais, São Paulo and Paraná (Ranga \& Silva 2002). According to Pennington (1997), Inga lanceifolia only occurs in Rio de Janeiro but its distribution was extended towards São Paulo. Ixora heterodoxa (Rubiaceae) occurs from Bahia to Rio Grande do Sul, in the coastal region (Anderson 1992). Maytenus schumanniana (Celastraceae), according to Carvalho-Okano (2005), occurs in Bahia, Espírito Santo, São Paulo and Paraná States, with the most samples in São Paulo and Paraná. Rhodostemonodaphne macrocalyx (Lauraceae) occurs from south of Bahia to Paraná (Baitello 2003). Tibouchina arborea (Melastomataceae) occurs on the coastal plain from Espírito Santo to São Paulo State according to Guimarães \& Oliveira (2009). Vernonia petiolaris (Asteraceae) occurs in Minas Gerais, Rio de Janeiro, São Paulo, Paraná and Santa Catarina (J. Semir unpublished data).

Despite the biological coherence of the data analysis, its interpretation must still be made carefully, since it is noteworthy that the distribution of those exclusive species is not restricted. Therefore the surveys are still not representative of the Brazilian Atlantic rain forest flora, and more floristic studies are necessary to improve the knowledge of this biome.

The results of the present study suggest that it is essential to preserve areas from the whole distribution of the Atlantic rain forest as its composition is quite complex along its range and it is not adequately represented by small localized areas.

Acknowledgements - We thank Capes for the grant received by the first author, the landowners of Fazenda Folha Larga, Mr. Max Ramos Lucas and Ms. Mariuza Lindemberg, for their inconditional support, G.J. Shepherd for the FITOPAC2.1 software, and the following taxonomists for the identifications: A.B. Martins (Melastomataceae), E. Lucas (Myrtaceae), I. Cordeiro (Euphorbiaceae), J.B. Baitello, P.L.R. Moraes and T.D.M. Barbosa (Lauraceae), J.E.C. Meireles (Fabaceae), J. Semir (Asteraceae), L.C. Bernacci (Myrsinaceae and Arecaceae), M. Moraes (Asteraceae), M. Sobral (Myrtaceae), S. Jung-Mendaçolli (Rubiaceae), S. Romaniuc Neto (Moraceae).

\section{References}

AIDAR, M.P.M., GODOY, J.R.L., BERGMANN, J. \& JOLY, C.A. 2001. Atlantic Forest succession over calcareous soil, Parque Estadual Turístico do Alto Ribeira - Petar, SP. Revista Brasileira de Botânica 24:455-469.

ANDERSON, L. 1992. A provisional checklist of neotropical Rubiaceae. Scripta Botanica Belgica 1:1-130.

APG. 2003. An update of the Angiosperm Phylogeny Group classification for the orders and families of flowering plants: APG II. Botanical Journal of Linnean Society 141:399-436.

ASSIS, M.A. 1999. Florística e caracterização das comunidades vegetais da Planície Costeira de Picinguaba, Ubatuba - SP. Tese de doutorado, Universidade Estadual de Campinas, Campinas.

BAITELLO, J.B. 2003. Lauraceae. In Flora Fanerogâmica do Estado de São Paulo (M.G.L. Wanderley, G.J. Shepherd, A.M. Giulietti \& T.S. Melhem, coords.). Fapesp/RiMa, São Paulo, v.3, p.149-223.

BENZECRI, P.J. 1992. Correspondence analysis handbook. Marcel Dekker, New York.

BERNACCI, L.C. \& JUNG-MENDAÇOLLI, S.L. 2000. Considerações taxonômicas e novas combinações em Ardisia Swartz (Myrsinaceae) do sudeste do Brasil. Acta Botanica Brasilica 14:243-249.

BRAZ, D.M., MOURA, M.V.L.P.M. \& ROSA, M.M.T. 2004. Chave de identificação para as espécies de Dicotiledôneas arbóreas da Reserva Biológica do Tinguá, RJ, com base em caracteres vegetativos. Acta Botanica Brasilica 18:225-240. 
BRUMMIT, R.K. \& POWELL, C.E. 1992. Authors of plant names. Royal Botanic Gardens, Kew.

CARVALHO-OKANO, R.M. 2005. Celastraceae. In Flora Fanerogâmica do Estado de São Paulo (M.G.L. Wanderley, G.J. Shepherd, T.S. Melhem \& A.M. Giulietti, coords.). Fapesp/RiMa, São Paulo, v.4, p.185-194.

CESAR, O. \& MONTEIRO, R. 1995. Florística e fitossociologia de uma floresta de Restinga em Picinguaba (Parque Estadual da Serra do Mar), Município de Ubatuba - SP. Naturalia 20:89-105.

CUSTODIO FILHO, A., NEGREIROS, O.C., DIAS, A.C. \& FRANCO, G.A.D.C. 1992. Composição florística do estrato arbóreo do Parque Estadual de Carlos Botelho, SP. Revista do Instituto Florestal 4:184-191.

EMBRAPA. 2006. Sistema Brasileiro de Classificação de Solos. Embrapa Solos, Rio de Janeiro.

GUIMARÃES, P.J.F. \& OLIVEIRA, C.M.S. 2009. Tibouchina. In Flora Fanerogâmica do Estado de São Paulo (M.G.L. Wanderley, G.J. Shepherd, T.S. Melhem \& A.M. Giulietti, coords.). Fapesp/Instituto de Botânica, São Paulo, v.6, p.127-149.

HENDERSON, A., GALEANO, G. \& BERNAL, R. 1995. Field guide to the palms of the Americas. Princeton University, New Jersey.

HILL, M.O. \& GAUCH, H.G. 1980. Detrended Correspondence Analysis: an improved ordination technique. Vegetatio 42:47-58.

IVANAUSKAS, N.M., MONTEIRO, R. \& RODRIGUES, R.R. 2001. Levantamento florístico de trecho de Floresta Atlântica em Pariquera-Açu, São Paulo, Brasil. Naturalia 26:97-129.

JOLY, C.A., AIDAR, M.P.M. \& KLINK, C.A. 1999. Evolution of the Brazilian phytogeography classification systems: implications for biodiversity conservation. Ciência e Cultura 51:331-348.

KOEPPEN, W. 1948. Climatologia: com um estudio de los climas de la terra. Fundo de Cultura Economica, México.

KURTZ, B.C. \& ARAÚJO, D.S.D. 2000. Composição florística e estrutura do componente arbóreo de um trecho de Mata Atlântica na Estação Ecológica Estadual do Paraíso, Cachoeiras de Macacu, Rio de Janeiro, Brasil. Rodriguesia 51:69-111.

LEITÃO FILHO, H.F. 1982. Aspectos taxonômicos das florestas do estado de São Paulo. Silvicultura em São Paulo 1:206-297.

LEITÃO FILHO, H.F. (coord.) 1993. Ecologia da Mata Atlântica em Cubatão. Universidade Estadual Paulista/ Universidade Estadual de Campinas, Campinas.

MAMEDE, M.C., CORDEIRO, I., ROSSI, L. MELO, M.M.R.F. \& OLIVEIRA, R.J. 2004. Mata Atlântica. In Estação Ecológica Juréia-Itatins, ambiente físico, flora e fauna (O.A.V. Marques \& W. Duleba, eds.). Holos, Ribeirão Preto, p.115-132.
MANTOVANI, W. 1992. A vegetação sobre restinga em Caraguatatuba, SP. Revista do Instituto Florestal 4:139144.

MARTINS, A.B., SEMIR, J., GOLDEMBERG, R. \& MARTINS, E. 1996. O gênero Miconia Ruiz and Pav. (Melastomataceae) no Estado de São Paulo. Acta Botanica Brasilica 10:267-316.

MARTINS, H.F. 1988. Duas novas espécies de Callisthene (Vochysiaceae). Bradea 5:149.

MELO, E. \& MARCONDES-FERREIRA, W. 2009. Polygonaceae. In Flora Fanerogâmica do Estado de São Paulo (M.G.L. Wanderley, G.J. Shepherd, T.S. Melhem \& A.M. Giulietti, coords.). FAPESP/Instituto de Botânica, São Paulo, v.6, p.169-193.

MORENO, M.R., NASCIMENTO, M.T. \& KURTZ, B.C. 2003. Structure and floristic composition of tree communities in two altitudinal zones in an Atlantic forest in the Imbé Region, RJ, Brazil. Acta Botanica Brasilica 17:371-386.

MUELLER-DOMBOIS, D. \& ELLENBERG, H. 1974. Aims and methods of vegetation ecology. John Wiley, New York.

MYERS, N., MITTERMEIER, R.A., MITTERMEIER, C.G., FONSECA, G.A.B. \& KENT, J. 2000. Biodiversity hotspots for conservation priorities. Nature 403:853858.

NEGRELLE, R.R.B. 2002. The Atlantic Forest in the Volta Velha Reserv: a tropical rain forest site outsite the tropics. Biodiversity and Conservation H:887-919.

OLIVEIRA FILHO, A.T. \& FONTES, M.A. 2000. Patterns of floristic differentiation among Atlantic Forests in southern Brazil and the influence of the climate. Biotropica 32:793-810.

PEIXOTO, G.L., MARTINS, S.V., SILVA, A.F. \& SILVA, E. 2004. Floristic survey of the tree layer in an area of Atlantic Rain forest in Serra da Capoeira Grande Environmental Protection Area, Rio de Janeiro State, Brazil. Acta Botanica Brasilica 18:151-160.

PENNINGTON, T.D. 1990. Flora Neotropica - Sapotaceae. The New York Botanical Garden, New York.

PENNINGTON, T.D. 1997. The genus Inga botany. The Royal Botanic Gardens, Kew.

PLANT NAMES PROJECT. 2004. The International Plant Names Index. http://www.inpi.org. (accessed 2004 Dec 12).

RANGA, N.T. \& SILVA, L.C. 2002. Boraginaceae. In Flora fanerogâmica da Ilha do Cardoso (M.R.F.M. Melo, F. Barros, S.A.C. Chiea, M. Kirizawa, S. Jung-Mendaçolli \& M.G. Wanderley, eds.). Instituto de Botânica, São Paulo, v.9, p.105-114.

RIBEIRO, M.C., METZGER, J.P., MARTENSEN, A.C., PONZONI, F.J. \& HIROTA, M.M. 2009. The Brazilian Atlantic Forest: How much is left, and how is the remaining forest distributed? Implications for conservation. Biological Conservation 142:1141-1153. 
RIZZINI, C.T. 1997. Tratado de fitogeografia do Brasil: aspectos ecológicos, sociológicos e florísticos. Âmbito Cultural Edições, Rio de Janeiro.

RODRIGUES, R.R. 2006. II Relatório Parcelas Permanentes. http://www.lerf.esalq.usp.br/parcelas. (accessed 2006 Jun 7).

ROTTA, E., BOEGER, M.R. \& GRODZKI, L. 1992. Levantamento florístico e fitossociológico de um trecho de Floresta Ombrófila Densa das terras baixas no Parque Estadual do Palmito, Paranaguá, PR. Boletim de Pesquisas Florestais 24/25:21-28.

SAMPAIO, D., SOUZA, V.C., OLIVEIRA, A.A., PAULASOUZA, J. \& RODRIGUES, R.R. 2005. Árvores da restinga: guia ilustrado para a identificação das espécies da Ilha do Cardoso. Neotropica, São Paulo.

SANCHEZ, M., PEDRONI, F., LEITÃO FILHO, H.F. \& CÉSAR, O. 1999. Composição florística de um trecho de floresta ripária na Mata Atlântica em Picinguaba, Ubatuba, SP. Revista Brasileira de Botânica 22:31-42.

SÃO PAULO - Secretaria de Estado do Meio Ambiente, 1996. Regulamentação da APA Cananéia-IguapePeruíbe: plano de gestão - unidade de gestão - ZEE preliminar. Secretaria do Meio Ambiente do Estado de São Paulo/Ibama, São Paulo.

SCUDELLER, V.V., MARTINS, F.R. \& SHEPHERD, G.J. 2001. Distribution and abundance of arboreal species in the Atlantic Ombrophilous Dense Forest in southern Brazil. Plant Ecology 152:185-199.

SHEPHERD, G.J. \& URBANETZ, C. 2010. FITOPAC2.1: Manual do usuário. Departamento de Biologia Vegetal, Universidade Estadual de Campinas, Campinas.

SILVA, A.F.S. \& LEITÃO FILHO, H.F. 1982. Composição florística e estrutura de um trecho de Mata Atlântica de Encosta no Município de Ubatuba (São Paulo, Brasil). Revista Brasileira de Botânica 5:43-52.

SILVA, F.C. 1994. Composição florística e estrutura fitossociológica da floresta tropical ombrófila da encosta Atlântica no Município de Morretes, estado do Paraná. Acta Biológica Paranaense 23:1-54.
SILVA, G.C. \& NASCIMENTO, M.T. 2001. Phytosociological studies in a remnant lowland forest on tertiary tabuleiros in the north Rio de Janeiro State. Revista Brasileira de Botânica 24:51-62.

SILVA, S.M. 1990. Composição Florística e Estrutura Fitossociológica de um Trecho de Floresta de Restinga na Ilha do Mel, Município de Paranaguá, PR. Dissertação de mestrado, Universidade Estadual de Campinas, Campinas.

SOLOMON, J. 2004. W³ Tropicos. http://www.mobot.org/ W3T/Search/vast.html (accessed 2004 Dec 12).

SUGIYAMA, M. 1998. Estudo de florestas da restinga da Ilha do Cardoso, Cananéia, São Paulo, Brasil. Boletim do Instituto de Botânica 11:119-159.

SZTUTMAN, M. \& RODRIGUES, R.R. 2002. Vegetational mosaic of contiguous forest area in a coastal plain, Campina do Encantado State Park, Pariquera-Açu, SP. Revista Brasileira de Botânica 25:161-176.

VELOSO, H.P., OLIVEIRA FILHO, L.C., VAZ, A.M.S.F., LIMA, M.P.M., MARQUETE, R. \& BRAZÃO, J.E.M. (orgs.) 1992. Manual técnico da vegetação brasileira. IBGE, Rio de Janeiro, v.1.

WANDERLEY, M.G.L., SHEPHERD, G.J. \& GIULIETTI, A.M. (coords.) 2002. Flora Fanerogâmica do Estado de São Paulo. Fapesp/Hucitec, São Paulo, v.2.

WANDERLEY, M.G.L., SHEPHERD, G.J., GIULIETTI, A.M. \& MELHEM, T.S. (coords.) 2003. Flora Fanerogâmica do Estado de São Paulo. Fapesp/RiMa, São Paulo, v.3.

WANDERLEY, M.G.L., SHEPHERD, G.J., MELHEM, T.S. \& GIULIETTI, A.M. (coords.) 2005. Flora Fanerogâmica do Estado de São Paulo. Fapesp/RiMa, São Paulo, v.4.

WANDERLEY, M.G.L., SHEPHERD, G.J., MELHEM, T.S. \& GIULIETTI, A.M. (coords.) 2007. Flora Fanerogâmica do Estado de São Paulo. Fapesp/Instituto de Botânica, São Paulo, v.5.

WANDERLEY, M.G.L., SHEPHERD, G.J., MELHEM, T.S. \& GIULIETTI,A.M. (coords.) 2009. Flora Fanerogâmica do Estado de São Paulo. Fapesp/Instituto de Botânica, São Paulo, v.6. 
